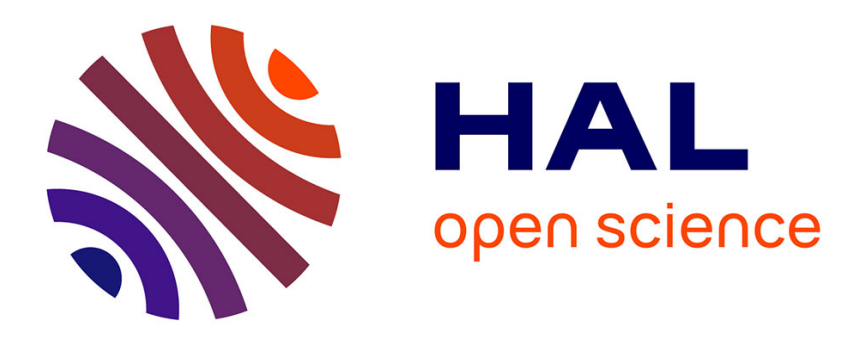

\title{
Transmission simultanée de voix et données
}

A. Durand, C. Galand

\section{To cite this version:}

A. Durand, C. Galand. Transmission simultanée de voix et données. Revue de Physique Appliquée, 1984, 19 (2), pp.121-126. 10.1051/rphysap:01984001902012100 . jpa-00245163

\section{HAL Id: jpa-00245163 https://hal.science/jpa-00245163}

Submitted on 1 Jan 1984

HAL is a multi-disciplinary open access archive for the deposit and dissemination of scientific research documents, whether they are published or not. The documents may come from teaching and research institutions in France or abroad, or from public or private research centers.
L'archive ouverte pluridisciplinaire HAL, est destinée au dépôt et à la diffusion de documents scientifiques de niveau recherche, publiés ou non, émanant des établissements d'enseignement et de recherche français ou étrangers, des laboratoires publics ou privés. 


\title{
Transmission simultanée de voix et données
}

\author{
A. Durand et C. Galand \\ IBM France, Centre d'Etudes et de Recherches, 06610 La Gaude, France
}

(Reçu le 3 juin 1983, révisé le 3 octobre, accepté le 19 octobre 1983)

\begin{abstract}
Résumé. - Après une revue des problèmes liés à la numérisation et à la compression du signal vocal, les auteurs présentent un exemple de codage à débit moyen (codage prédictif à excitation par la bande de base - 7200 bit/s) et discutent son insertion dans un environnement télématique.
\end{abstract}

\begin{abstract}
After an overview of the problems of digitization and compression of the voice signal, the authors present a medium bit rate coding algorithm (Voice Excited Predictive Coding - $7200 \mathrm{bps}$ ) and discuss its use into teleprocessing environment.
\end{abstract}

\section{De la voix analogique à la voix numérisée.}

La représentation numérique de la parole s'est imposée au fil de l'évolution des techniques de transmission avec l'apport d'une transmission théoriquement sans dégradation par la distance; en fait légèrement dégradée par les erreurs de ligne mais d'une façon calculable. Des avantages additionnels sont apportés par le stockage numérique et les possibilités de chiffrement pour la protection des communications confidentielles.

Nous nous intéressons ici aux applications réalisant analyse de la parole et synthèse de la parole en temps réel, simultanément ou non, à l'exclusion des applications de synthèse à partir d'un texte ou d'un vocabulaire élaboré en usine.

1.1 EChantillonnage et Quantification. - Deux grandes familles de techniques sont aujourd'hui largement utilisées.

Les modulations Delta ont eu longtemps l'avantage $\mathrm{du}$ codec plus simple et gardent l'avantage d'une meilleure résistance aux erreurs de transmission. Le meilleur exemple actuel est l'échantillonnage à $32 \mathrm{kHz}$ avec la technique de la pente variable en continu (en anglais CVSD) produisant un signal à $32 \mathrm{kbit} / \mathrm{s}$ et fournissant une qualité de reproduction très satisfaisante. Toutes les $31,25 \mu$ s un échantillon de 1 bit est produit, et il n'est pas nécessaire d'aligner le flot de bits sur une autre base de temps.

La Modulation par Impulsions Codées (MIC, en anglais PCM) a l'avantage d'une normalisation internationale (Loi A du CCITT, loi Mu en Amérique du Nord) et est désormais supportée par des codecs intégrés produits en grandes séries. L'échantillonnage à $8 \mathrm{kHz}$ et l'échantillon de 8 bits produisent un signal à $64 \mathrm{kbit} / \mathrm{s}$ fournissant une reproduction de qualité dite « transparente ». Toutes les $125 \mu$ s un échantillon de 8 bits est produit, il est nécessaire dans la transmission de traiter chaque échantillon comme un tout et d'effectuer une transmission en mode "caractère" ou avec synchronisation de trame. Ce type de numérisation est à la base des algorithmes de compression envisagés ci-dessous. Son inconvénient principal est l'exigence d'une bande passante bien supérieure à celle de la téléphonie analogique ce qui reste coûteux pour les transmissions sur de grandes distances.

\subsection{Algorithmes de Compression. - Pour diminuer} le volume d'information à transmettre ou à stocker on a recours à diverses méthodes de compression. Dans la réalisation pratique on est amené à un compromis entre la qualité du résultat, le débit résultant et les exigences de l'algorithme en complexité du programme et en puissance de calcul. Il est souhaitable de rester dans la limite d'une carte à microprocesseur.

Pour les débits situés entre $32 \mathrm{kbit} / \mathrm{s}$ et $16 \mathrm{kbit} / \mathrm{s}$ on recherche une qualité " transparente" sinon aux mesures physiques du moins à l'appréciation subjective. L'activité de recherche est importante et alimentée par la nécessité d'augmenter le trafic possible sur les satellites de communications. Un standard pourrait émerger basé sur les techniques de codage différentiel adaptatif ou MICDA (en anglais ADPCM).

De 16 à $4,8 \mathrm{kbit} / \mathrm{s}$ on est à la recherche de la qualité " communication" ou "sub-téléphonique", on ne saurait prétendre à la transparence absolue mais à 
une qualité satisfaisant aux besoins courants de la communication téléphonique. Nous nous intéresserons particulièrement à ce type de compression qui correspond au domaine des vitesses moyennes de transmission en informatique sur ligne louée standard, et qui permet un multiplexage sur lignes à grande vitesse.

De 4800 à 800 bit/s on est dans le domaine de la qualité "synthétique " avec une dégradation sensible du message transmis et dans les meilleurs cas une bonne identification du locuteur. L'intérêt de ces transmissions à bas débit est cependant grand pour des messages de service ou des transmissions militaires.

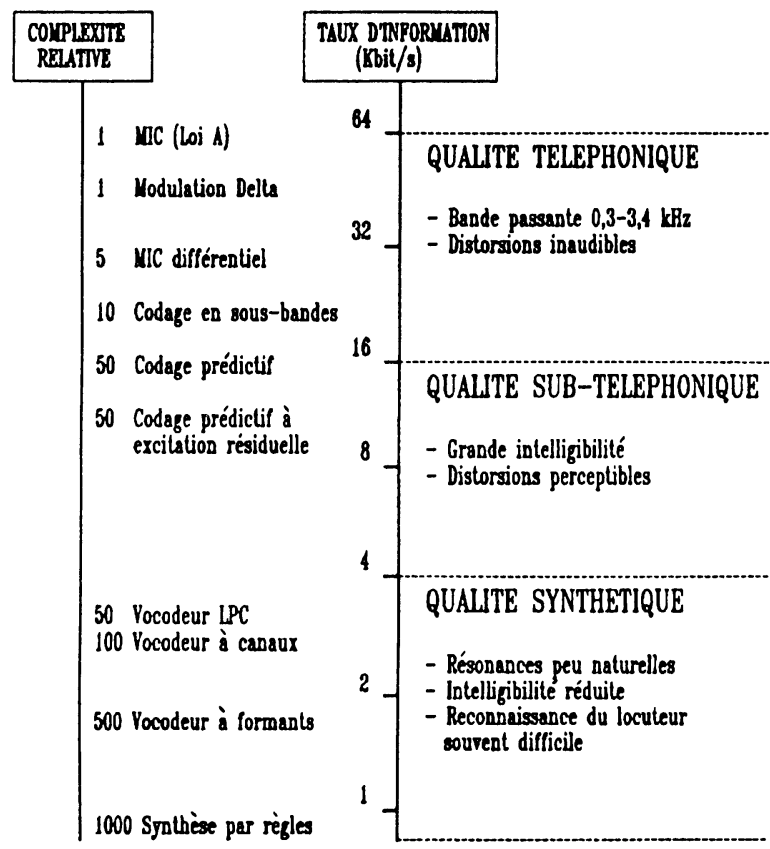

Fig. 1. - Méthodes de compression.

[Compression algorithms.]

1.3 LES PARAMÈTRES TEMPORELS DE LA CONVERSATION. - Dans le cas même du monologue, on identifie assez facilement une succession de " silences" et de " paroles». Des « silences » il est tentant de ne transmettre ou de stocker que l'information "durée ". Dans le cas du stockage de la parole, la seule donnée à prendre en considération est le pourcentage de " parole». Celui-ci varie assez largement avec le schéma de détection d'activité voix (en anglais VAD).

Dans le cas d'une transmission multiplexée ou par paquets, il y a lieu de considérer également la répartition statistique des "silences" et des "paroles". Une étude déjà ancienne de P. T. Brady [1] a servi longtemps de référence, le très grand mérite en est d'étudier des conversations réelles et de définir les variations du taux d'activité en fonction du détecteur d'activité choisi; toutefois la variation portait seulement sur un niveau de référence et le système de détection s'appliquait plus particulièrement à un environnement de multiplexage analogique.
Une étude plus récente de J. G. Gruber [2] considère les paramètres et ajuste un modèle mathématique à des résultats mesurés, ce qui permet la simulation d'un grand nombre de conversations après avoir effectué quelques mesures sur le système étudié.

Il est à noter que les durées moyennes des « paroles » et "silences" varient largement avec le schéma de détection d'activité. Brady donne un exemple $1300 /$ $1900 \mathrm{~ms}$ et Gruber un exemple 169/123 ms.

Les caractéristiques particulières du dialogue, alternance des locuteurs et intervention de l'auditeur ont fait l'objet d'études liées aux suppressions d'échos en particulier par Norwine [3], puis de tentatives de modélisation par Gustafson [4] qui introduit une séparation entre les courts silences et les pauses, et par Jaffe [5] qui utilise les chaînes de Markov.

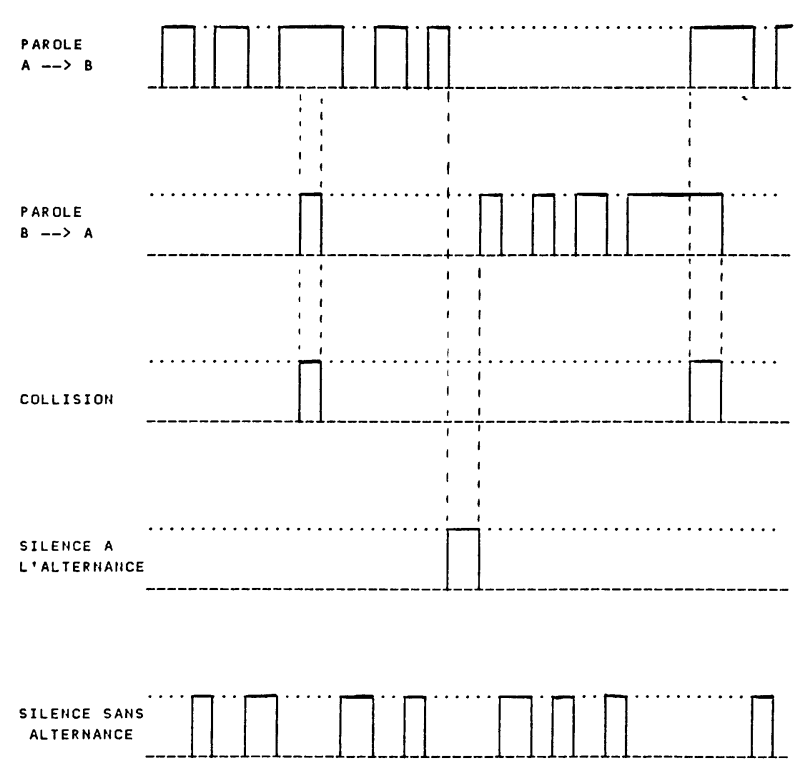

Fig. 2. - Paramètres temporels.

[Temporal parameters.]

Les divers modèles sont d'une grande utilité pour évaluer les compromis à trouver entre détection d'activité, délais, échos, etc. Ils permettent aussi d'étudier des cas défavorables peu observables en pratique et réduisent le nombre de mesures physiques à effectuer.

Selon les cas on représentera le dialogue avec toutes les transitions et interruptions ou on représentera un monologue avec une extrapolation au dialogue.

1.4 Détection D’ACTIVITÉ. - Elle peut se faire, classiquement par le niveau, sur une période plus ou moins longue; cette méthode analogique est très facile à utiliser en numérique. On peut aussi l'intégrer à l'algorithme de compression et la lier à la détection d'une modulation ou d'une fréquence.

Dans le cas de doute sur le début d'un silence ou pour éviter un effet de coupure audible on a souvent recours à un trainage pouvant atteindre $200 \mathrm{~ms}$.

Dans les systèmes d'audio-messagerie on peut aussi 

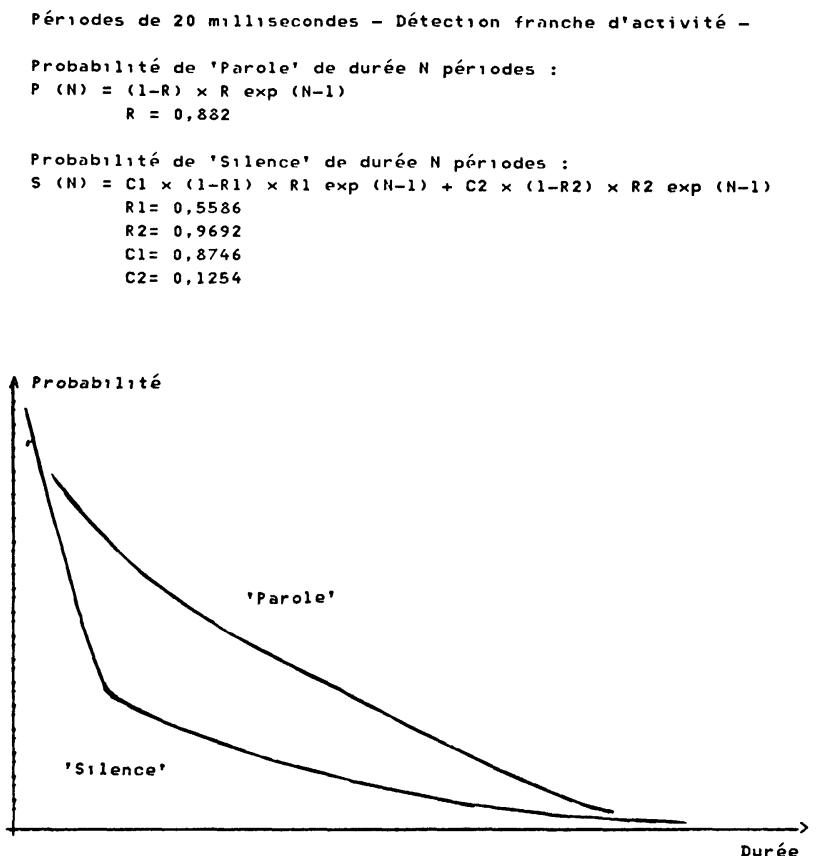

Fig. 3. - Modèle élémentaire.

[Basic model.]

restituer les derniers instants de "présumé silence " avant le début de la parole.

Dans tous les cas il s'agit de pallier les imperfections de la détection d'activité ou de la transmission par une augmentation du volume de données.
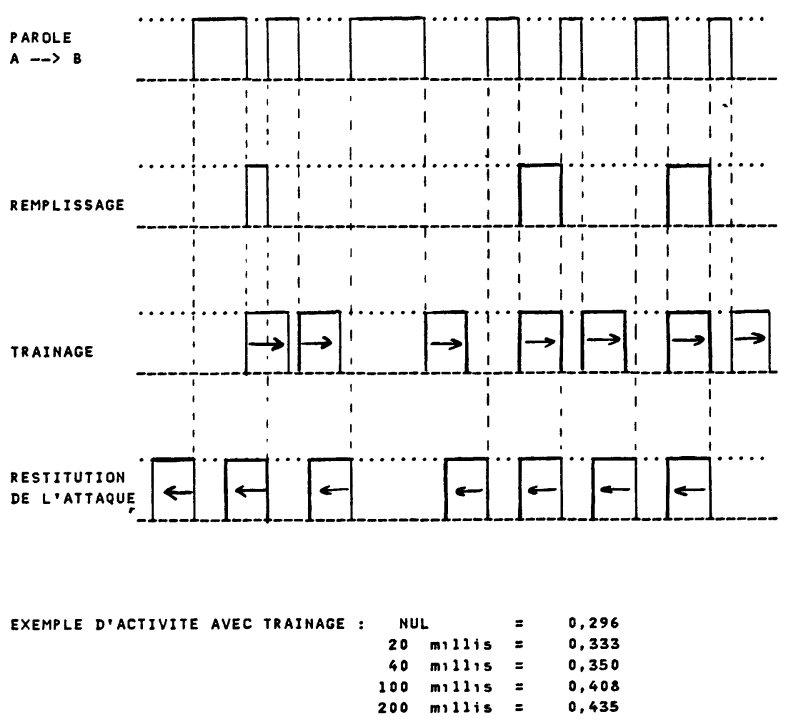

Fig. 4. - Trainage, remplissage et restitution.

[Hangover, fill-in and reconstitution.]

1.5 LES DIVERS DÉLAIS. - On peut suivre une chaîne complète de transmission pour analyser l'apparition des délais.

- Le premier délai introduit dans la transmission numérique de la parole est le délai d'échantillonnage et de transmission en série par le codec qui reste de l'ordre de quelques microsecondes.
- Les délais liés à l'algorithme de compression viennent ensuite :

- Accumulation de plusieurs échantillons pour analyse globale, par exemple 160 échantillons MIC pour $20 \mathrm{~ms}$ de parole.

- Durée du traitement incluant le temps de calcul proprement dit et éventuellement des délais de propagation dus au filtrage numérique. Ce délai peut donc varier de quelques microsecondes à plus de $50 \mathrm{~ms}$.

- Les délais liés à la transmission.

- Délai de trame ou de paquet à l'émission.

- Le temps de transmission physique, fixe ou variable. Le délai variable, lié à la nature des réseaux de transmission par paquet se paie par la nécessité de transmettre une horloge et par une attente supplémentaire à l'arrivée pour lisser les variations.

- Délai de trame ou de paquet à la réception.

- Les délais liés à l'algorithme de synthèse.

- La durée du calcul proprement dit.

- Le stockage des résultats de la synthèse.

- La transmission série au codec et la restitution.

Le CCITT considère que les délais de $300 \mathrm{~ms}$ sont toujours acceptables si les échos sont contrôlés de façon appropriée [6]; par contre les résultats portant sur des délais de l'ordre de $600 \mathrm{~ms}$ (correspondant par exemple à deux circuits par satellite en série) ne sont pas totalement en concordance et il est recommandé d'être prudent pour les délais supérieurs à $300 \mathrm{~ms}$.

Certains auteurs estiment que si l'écho est parfaitement contrôlé le retard fixe ne devient inacceptable que bien au-delà de la seconde [7].

1.6 LES ÉCHOS ET LES MÉCANISMES DE CONTRÔLE. Il s'agit ici de contrôler les différents échos électriques introduits surtout par les passages 2 fils- 4 fils. L'effet en est d'autant plus sensible que le temps de propagation est grand [8].

Au-delà de $25 \mathrm{~ms}$ le CCITT recommande un dispositif de contrôle de l'écho. Les deux types classiques sont le suppresseur d'écho et l'annuleur d'écho. Dans les applications avec compression numérique de la voix introduisant un délai important, il semble nécessaire d'utiliser un annuleur qui peut être intégré au mécanisme de compression.

Les performances exigées sont particulièrement élevées pour les transmissions par satellites $(270 \mathrm{~ms})$ ou pour les transmissions dans un réseau de paquets (délai variable).

\subsection{LA RÉSISTANCE DES ALGORITHMES AUX ERREURS} DE TRANSMISSION. - En transmission MIC non multiplexée une erreur sur un bit rend un échantillon de 8 bits erroné sans autre conséquence si la synchronisation est conservée.

En transmission de voix comprimée un bit erroné peut affecter un grand nombre d'échantillons.

Dans le cas d'une transmission multiplexée, par 
exemple en paquets, l'erreur est détectée au niveau du paquet et en l'absence de code autocorrecteur le paquet entier peut être considéré comme nul. Ceci limite le nombre de bits par trame ou par paquet et oriente le choix du mode de transmission sur un support donné.

\subsection{Multiplexage analogiQue et NuméRiQue. -} Le principe de base est de ne pas transmettre les silences et de tirer parti de la loi des grands nombres pour accepter un nombre de communications supérieur à la capacité théorique du canal utilisé. On doit aussi transmettre les changements d'affectation des voies physiques aux conversations.

Les administrations des PTT et les compagnies téléphoniques peuvent ainsi doubler le nombre de communications acheminées par certains canaux analogiques.

Avec les techniques de multiplexage numérique et sur des faisceaux déjà importants on peut envisager un triplement de la capacité [9]. De nombreuses études comparent les mérites respectifs des schémas en trame périodique, en paquets ou hybrides. On peut citer Coviello [10] et Weinstein [11] ainsi qu'un article de synthèse de Gruber [7] comportant une abondante bibliographie.

1.9 Les CRITÈres DE QUALITÉ. - On peut distinguer les critères classiques liés à la transmission proprement dite et les critères liés au multiplexage.

Pour le premier groupe et en ce qui concerne le MIC, le CCITT décrit tous les facteurs mesurables (volume, bruit de circuit, distorsions, délais, échos...) qui affectent la qualité téléphonique, mais les résultats de ces mesures ne sont pas utilisés pour définir un critère absolu de qualité, mais seulement pour prévoir si un système de transmission sera satisfaisant à l'oreille. Le seul véritable critère est subjectif et des méthodes d'évaluation sont recommandées [6].

Pour certaines applications de multiplexage analogique on a considéré qu'il était acceptable de perdre jusqu'à 5\% de la conversation. Le niveau suivant a été de s'intéresser à la répartition des coupures, et Campanella [12] a considéré qu'une probabilité de coupure de plus de $50 \mathrm{~ms}$ inférieure à $2 \%$ est acceptable.

Dans le cas de compression de la voix à débit variable comme suggéré par Dubnowski [13] ou Goodman [14] il y a lieu de définir des critères plus élaborés prenant en compte la durée et la gravité de la perte de débit.

\section{Un exemple de numérisation de la parole.}

Nous donnons ici un exemple de codage à débit moyen utilisant une technique de codage prédictif à excitation par la bande de base (en anglais Voice Excited Predictive Coding ou VEPC) proposée en 1978 [16] et expérimentée à différents débits. Une description détaillée se trouve en [15].
2.1 Algorithme. - Pour un bloc d'échantillons correspondant à $20 \mathrm{~ms}$ on détermine d'abord les coefficients de corrélation partielle (PARCOR). On calcule ensuite le signal résiduel et on prend en compte séparément les fréquences inférieures à $1000 \mathrm{~Hz}$ (ou bande de base) et les fréquences plus élevées.

Dans la bande de base on effectue un codage en sous-bandes grâce à des filtres numériques dits Filtres Miroirs en Quadrature (en anglais QMF). On utilise 6 filtres et le nombre de bits affectés à chaque sousbande est optimisé en permanence.

Pour la bande de haute fréquence on ne retient que la quantification de l'énergie du signal résiduel.

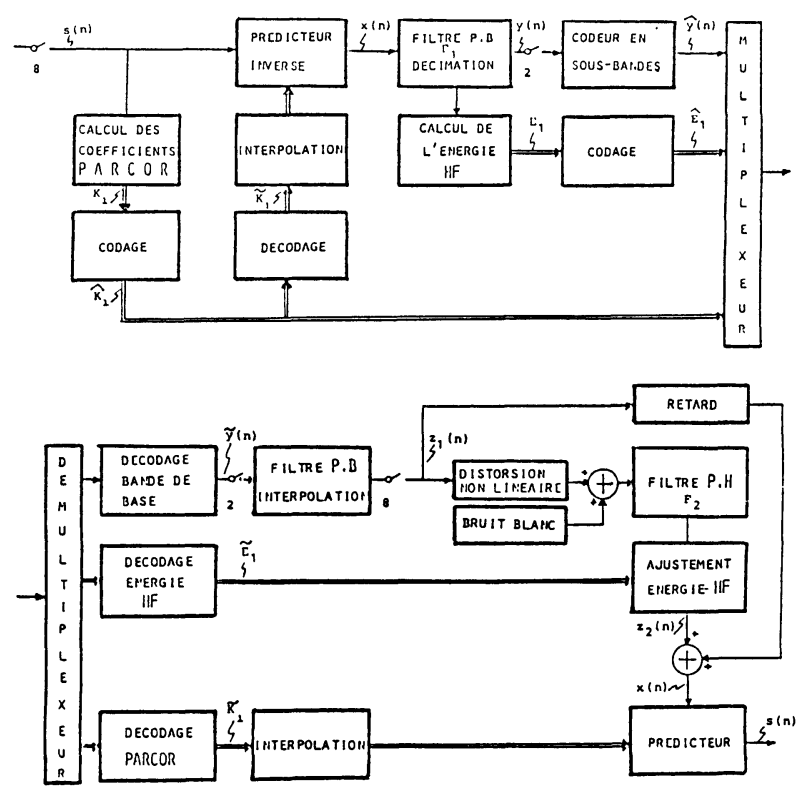

Fig. 5. - Algorithmes VEPC.

[VEPC algorithms.]

2.2 Critères De Choix. - La vitesse de $7200 \mathrm{bps}$ nous semble un bon compromis entre les performances et la qualité. Elle est compatible avec les équipements courants de transmission et traitement de données. Elle se prête bien au multiplexage sur ligne à grande vitesse (de 16 à 20 communications). Les algorithmes demandent une puissance voisine de 2.5 millions d'instructions par seconde (virgule fixe) pour l'analyse et un peu moins pour la synthèse. Les délais d'accumulation des échantillons, de traitement numérique et de restitution restent inférieurs à $100 \mathrm{~ms}$.

Dans la réalisation pratique les 144 bits transmis toutes les $20 \mathrm{~ms}$ sont affectés comme suit :

104 pour la bande de base dont 24 pour les caractéristiques des sous-bandes et 80 pour les valeurs.

8 pour l'énergie haute fréquence.

28 pour les coefficients PARCOR.

4 pour des informations de contrôle.

2.3 ECOUTE COMPARATIVE. - Le codeur VEPC a été évalué, sur le plan de la perception auditive, à l'aide 
de tests de jugement par paire [15]. Les tests comparatifs révèlent que le résultat est meilleur qu'avec la modulation Delta à $24 \mathrm{kbit} / \mathrm{s}$ ou qu'avec le codage par transformée à $16 \mathrm{kbit} / \mathrm{s}$.

Le rapport signal sur bruit équivalent se situe entre 18 et $21 \mathrm{~dB}$.

On notera cependant qu'un résultat supérieur est obtenu par un codeur en sous-bandes à $16 \mathrm{kbit} / \mathrm{s}$, un codeur prédictif adaptatif à $16 \mathrm{kbit} / \mathrm{s}$ ou un codeur différentiel avec quantification adaptative à $24 \mathrm{kbit} / \mathrm{s}$.

\section{Transmission de voix et données.}

3. 1 LA VOIX DANS L'ENVIRONNEMENT INFORMATIQUE. - Il s'agit ici de communication parlée limitée à l'intérieur d'une entreprise ou d'une application informatique.

3.1.1 Qualités requises. - Si la transparence absolue du procédé de numérisation n'est pas une nécessité, il n'en reste pas moins vrai qu'une très bonne qualité téléphonique est exigée. Une forte perte d'intelligibilité n'est pas admissible. La reconnaissance du locuteur (de tous les locuteurs) ne suffit pas et doit s'accompagner de la reconnaissance des intonations. De plus les tests subjectifs doivent permettre d'assurer que le système est " agréable " à l'écoute. La mise en service de nouvelles applications ou la réduction du coût d'applications existantes ne sera un succès que si l'utilisateur n'a pas l'impression d'en payer le prix dans ses conditions de travail.

3.1.2 Priorités. - On distingue ici le dialogue en temps réel et le monologue enregistré.

Dans le dialogue temps réel, le délai total est le point le plus critique et le contrôle de l'écho est très important. Le trainage peut pallier aux problèmes de durée variable de transmission.

Dans le monologue enregistré, on cherche une réduction du volume et on fait une optimisation poussée du mécanisme de détection d'activité, on peut remplacer le trainage par un remplissage sélectif et effectuer une restitution de l'attaque des mots. La durée des silences doit être enregistrée pour restitution.

3.1.3 Transmission et routage. - Dans la mesure où la voix enregistrée est destinée à rester dans un cadre informatique il n'y a pas de problème particulier. $\mathrm{Si}$ en sortie on passe à travers un autocommutateur privé, il est nécessaire de prévoir un raccordement avec appel automatique et une gestion des numéros d'appel.

3. 2 UN EXEMPLE ÉLÉMENTAIRE DE TRANSMISSION SIMULTANÉE. - Une ligne louée 4 fils de qualité normale peut transmettre une conversation téléphonique, en cours d'activité l'utilisation moyenne de la bande passante ne dépasse pas $35 \%$.

La même ligne reliant un terminal interactif intelligent à un ordinateur central à travers un modem full- duplex à 9600 bps sera aussi sans doute faiblement utilisée.

La même ligne partagée statiquement dans les rapports 7 200/2 400, $4800 / 4800$ ou $2400 / 7200$ entre voix et données verra une des applications pénalisée.

Dans un partage dynamique de la ligne on sacrifiera 400 bps pour le contrôle. La voix ne sera pas pénalisée. Les données auront un débit maximum de 9200 bps, un débit minimum de 2000 bps et un débit moyen supérieur à 6600 bps.

3. 3 RÉSEAUX VOIX ET DONNÉES. - Un grand nombre de réseaux en expérimentation ou en cours d'étude utilisent toutes les variations possibles de la commutation de circuits pure à la commutation de paquets [7].

Les études de trafic donnent l'avantage aux circuits pour les longs messages et aux paquets pour les messages courts; la notion de longueur étant liée au pourcentage de la bande passante totale demandée par une seule communication. Ainsi la voix codée à $64 \mathrm{kbit} / \mathrm{s}$ s'adapte mal aux réseaux de paquets existants. La compression et l'adoption d'un mode de transmission plus spécifique sont une solution.

L'extrapolation de l'exemple élémentaire ci-dessus à une ligne à $64 \mathrm{kbits} / \mathrm{s}$ permet de supporter une quinzaine de conversations et des données à priorité inférieure selon un schéma de paquets composites comportant :

- Un fanion si la ligne ne comporte pas de signal de synchronisation.

- Un en-tête donnant la répartition de l'information entre les « sessions " voix ou données et les informations de contrôle.

- Jusqu'à huit blocs pour les "sessions » voix en activité.

- Une quantité variable d'information pour les sessions données en cours.

- Eventuellement un code détecteur d'erreurs.

\section{Evolution en cours et conclusions.}

L'évolution, très rapide au cours de ces dernières années, de la technologie des composants électroniques et des transmissions numériques ouvre la voie à de nouvelles applications dans le cadre des réseaux en cours de définition.

4.1 Processeurs de SIGNal. - Ces microprocesseurs privilégient la capacité de calcul aux dépens des autres fonctions des microprocesseurs d'usage général. Pour les calculs rapides et complexes en temps réel, la multiplication est généralement câblée pour durer un seul cycle et des opérations sont parfois spécialement définies pour le calcul des transformées de Fourier.

Les processeurs affectés à une seule application prédéfinie coexistent avec les processeurs programmables. Les modèles actuellement commercialisés 
effectuent une opération en 200 ou 250 ns. Si ces vitesses sont déjà convenables pour le traitement de la parole en temps réel, il y a lieu d'espérer une augmentation continue des performances et une baisse des coûts élargissant le domaine d'application.

4.2 Supports DE TRANSMisSion. - Les satellites de communication en service ou en projet, offrent des canaux permanents ou commutés à 32,56 ou $64 \mathrm{kbit} / \mathrm{s}$ qui se prêtent bien à l'insertion de la voix dans le trafic de données.

Les fibres optiques vont rapidement équiper les autoroutes de la transmission numérique. Le raccordement direct de l'abonné suivra. De nouveaux services seront offerts aux usagers en particulier le Réseau Numé- rique à Intégration de Service (RNIS ou en anglais ISDN).

4. 3 LA COMPRESSION DE LA VOIX. - Une standardisation internationale des débits à $32 \mathrm{kbit} / \mathrm{s}$ (ADPCM) est prévue dès cette année, elle devrait précéder une standardisation à $16 \mathrm{kbit} / \mathrm{s}$ pour réduire la demande de bande passante. Le codage à débit inférieur a un grand avenir pour les applications particulières où il existe une limitation des capacités de transmission ou de stockage. L'association de la compression numérique de la parole et des méthodes de l'informatique et de la télématique doit permettre de nouvelles applications supportées par des composants de plus en plus puissants à des coûts de plus en plus modérés.

Bibliographie

[1] Brady, P. T., A Statistical analysis of On-Off Patterns in 16 Conversations, Bell System Tech. J. 47 January 1968.

[2] Gruber, J. G., A Comparison of Measured and Calculated Speech Temporal Parameters Relevant to Speech Activity Detection, IEEE Trans. Commun., 30, No. 4 (1980).

[3] Norwine, A. C., Murphy, O. J., Characteristics Time Intervals in Telephonic Conversations, Bell System Tech. J. 17 (1938).

[4] Gustafson, H. W., Model for the Analysis of Talkspurt and Silence Durations in Conversational Interaction, Proceedings 77th Annual Convention APA, 1969.

[5] JAFFe, J., Feldstein, S., Cassota, L., Markovian Models of Dialogic Time Patterns, Nature (1967).

[6] CCITT, Livre Jaune, Volume 5.1, Edition 1981.

[7] Gruber, J., Performances Considerations for Integrated Voice and Data Networks, Computer Comm. 4, No. 3 (1981).

[8] ERdreich, M., Le Phénomène de l'Echo dans les Réseaux de Télécommunications, Commutation et Transmission, 4, 1981.

[9] Adoul, J. P. et DaAboul, F., Digital TASI Generalization with Voiced/Unvoiced Discrimination for
Tripling T1 Carrier Capacity, ICC 77, Chicago (1977).

[10] Coviello, G. J., Comparative discussion of Circuit, vs. Packet-Switched Voice, IEEE Trans. Commun. 27, No. 8 (1979).

[11] Weinstein, C. J., Malpass, M. L. and Fisher, M. J., Data Traffic Performances of an Integrated Circuit and Packet-Switched Multiplex Structure, IEEE Trans. Commun. 28, No. 6 (1980).

[12] Campanella, S. J., Digital Speech Interpolation Techniques, NTC78, Birmingham (1978).

[13] Dubnowski, J. J. and Crochiere, R. E., Variable Rate of Speech, Bell System Tech. J. 58 No. 3 (1979).

[14] Goodman, D. J., Embedded DPCM for Variable Rate Transmission, ICC 80 Seattle (1980).

[15] Galand, C., Thèse de Docteur es Sciences Physiques, Codage en sous-bandes : Théorie et application à la compression numérique du signal de parole, Université de Nice (1983).

[16] Esteban, D., Galand, C., Mauduit, D., Menez, J., 9.6/7.2 kbps Voice Excited Predictive Coder (VEPC), IEEE ICASSP, Tulsa (1978). 